\title{
Manganese-based Nanoplatform as Metal Ion-Enhanced ROS Generator for Combined Chemodynamic/Photodynamic \\ Therapy
}

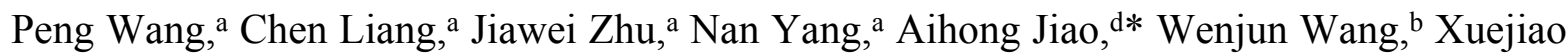
Song, ${ }^{\text {a* }}$ Xiaochen Dong ${ }^{\mathrm{a}, \mathrm{c} *}$

aKey Laboratory of Flexible Electronics (KLOFE) \& Institute of Advanced Materials (IAM), Nanjing Tech University (NanjingTech), 30 South Puzhu Road, Nanjing 211800, China.

E-mail:iamxcdong@njtech.edu.cn; xjsong@njtech.edu.cn

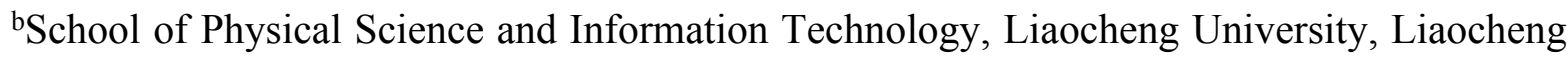
252059, China.

${ }^{c}$ School of Chemistry and Materials Science, Nanjing University of Information Science \& Technology, Nanjing 210044, China

${ }^{\mathrm{d}}$ Department of Chemotherapy, Yuhuangding Hospital, Yantai, Shandong 264000, China E-mail: jiaoaihong@126.com 


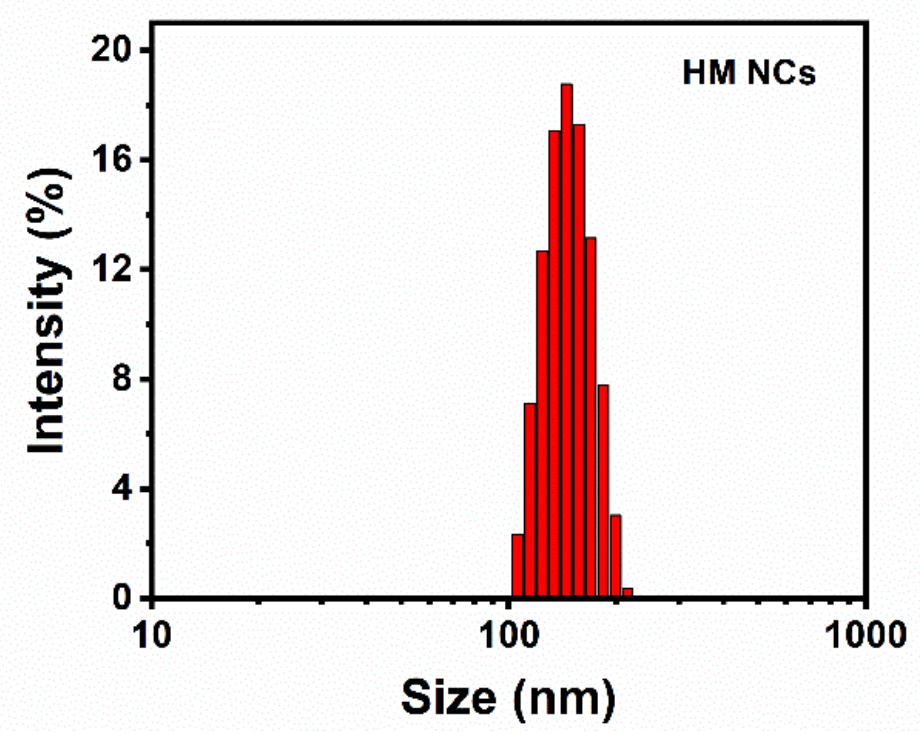

Figure S1. The size distribution of HM NCs.
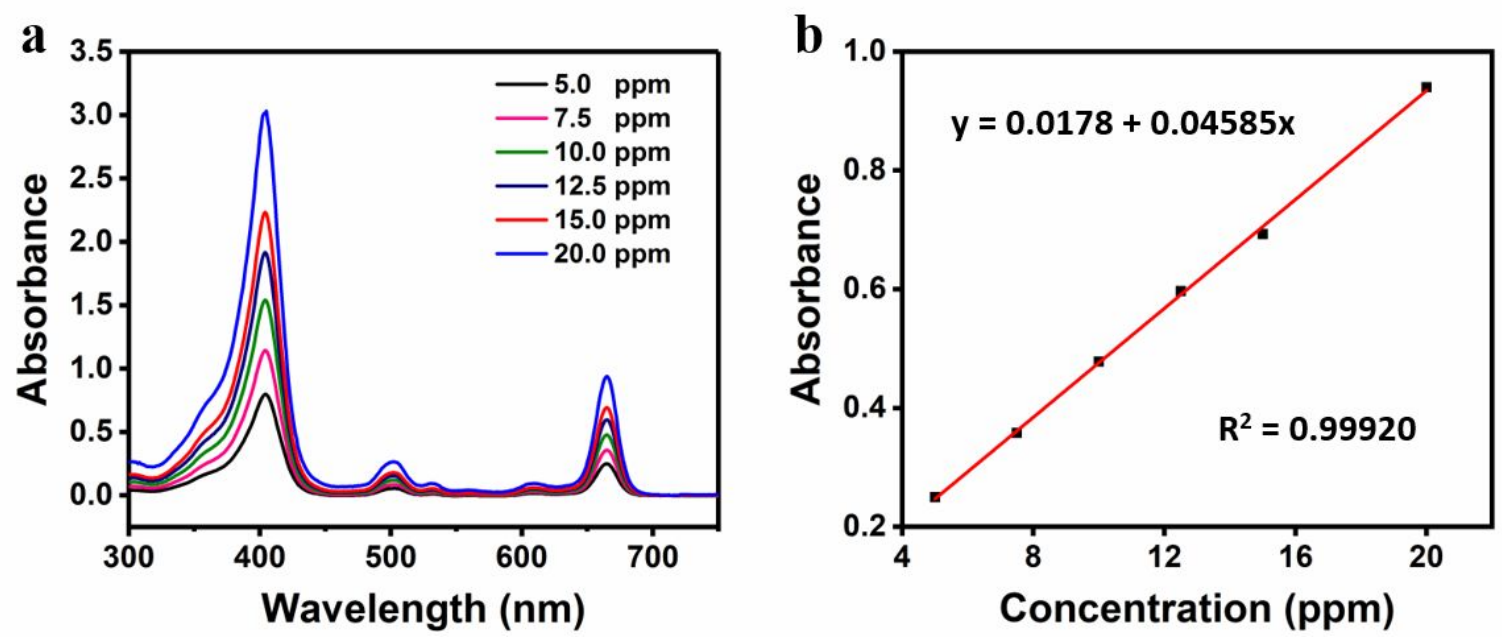

Figure S2. (a) UV-vis spectra of Ce6 dispersed in DMSO at various concentrations. (b): Lambert-Beer law absorbance plot for absorption at $404 \mathrm{~nm}$. 


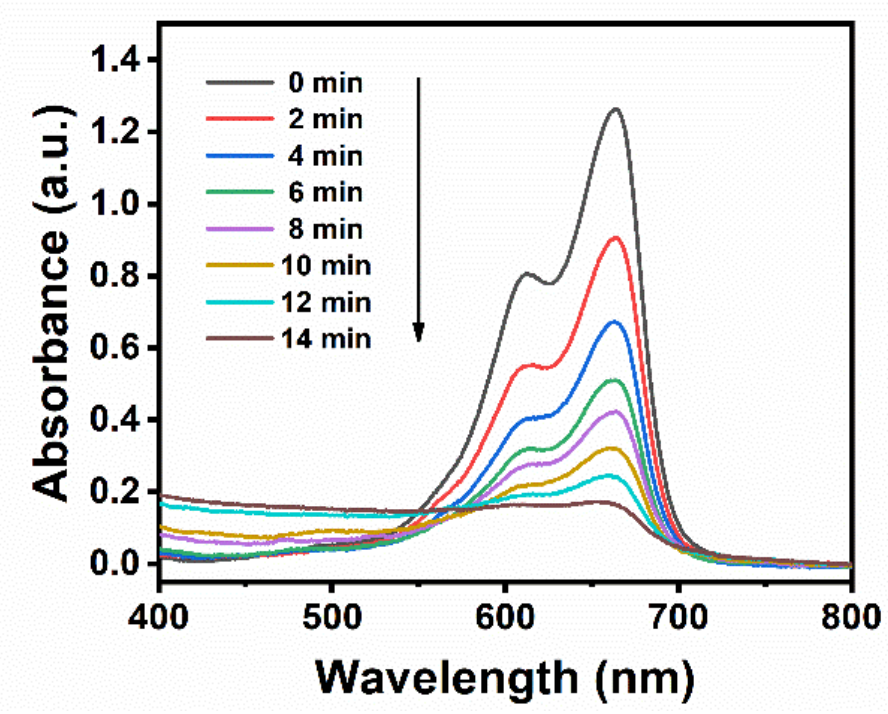

Figure S3. The degradation of MB induced by HM NCs with time.

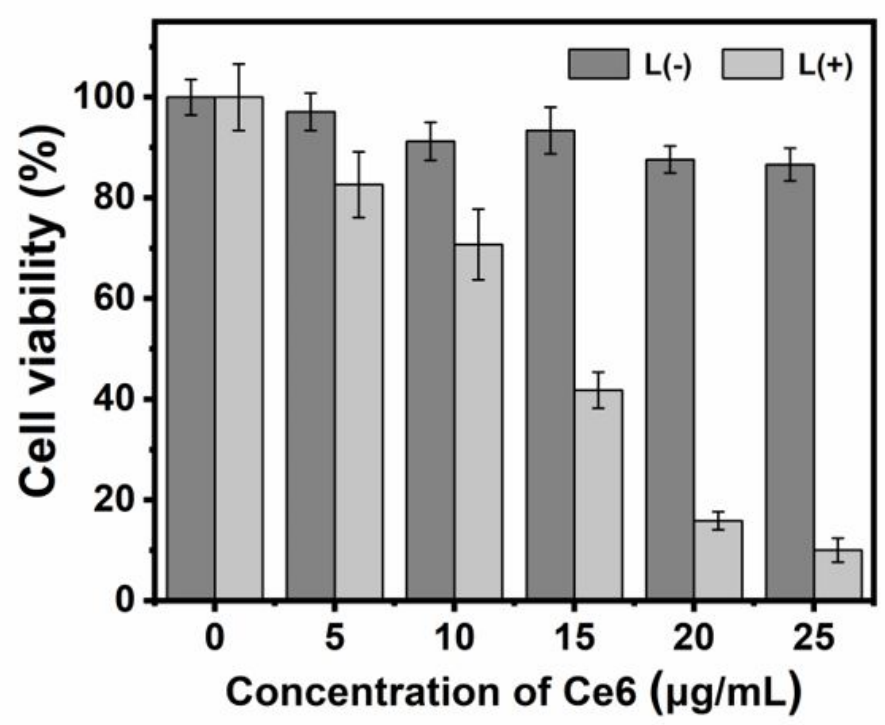

Figure S4. MTT assay of Hela cells incubated with HMCP NCs with or without irradiation. 


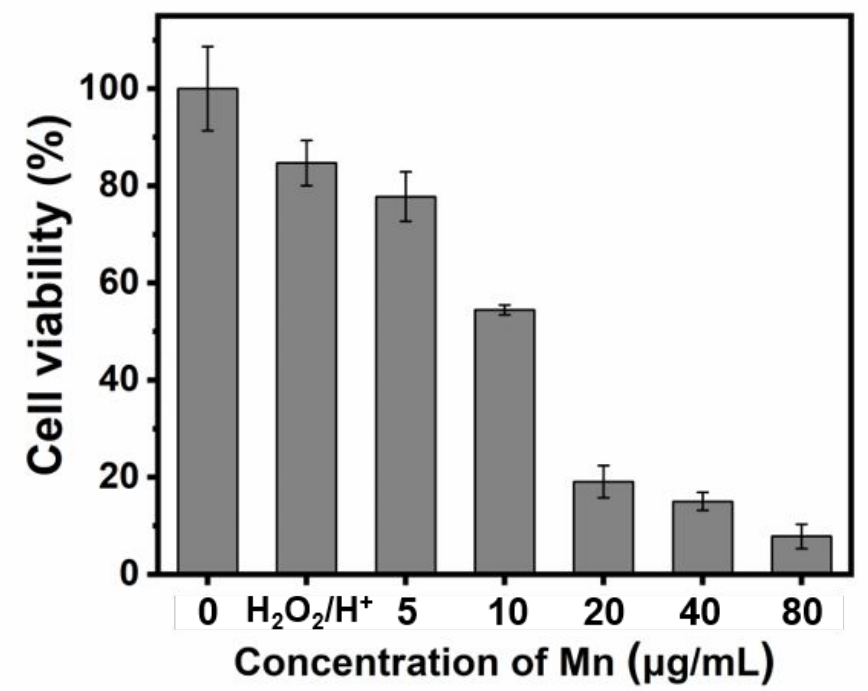

Figure S5. MTT assay of Hela cells incubated with HM NCs in the acidic medium containing $\mathrm{H}_{2} \mathrm{O}_{2}$.
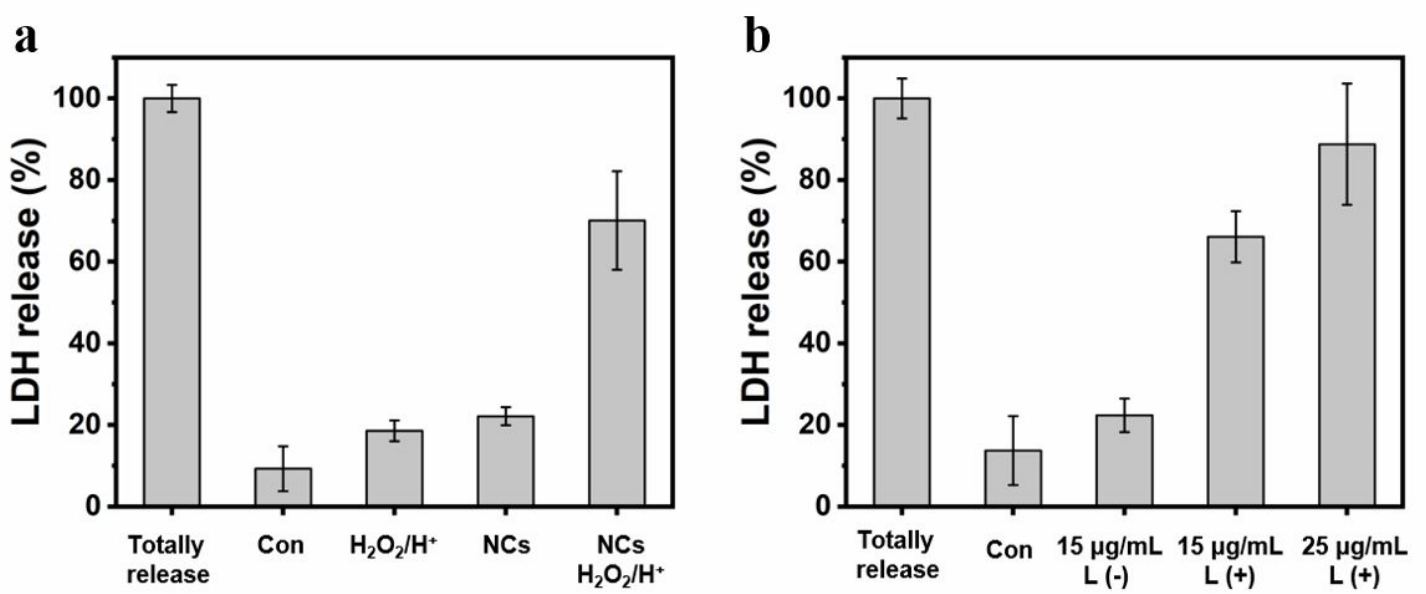

Figure S6. (a) LDH release assay in different medium. (b) LDH release assay of Hela cells incubated with HMCP NCs under different treatments. 


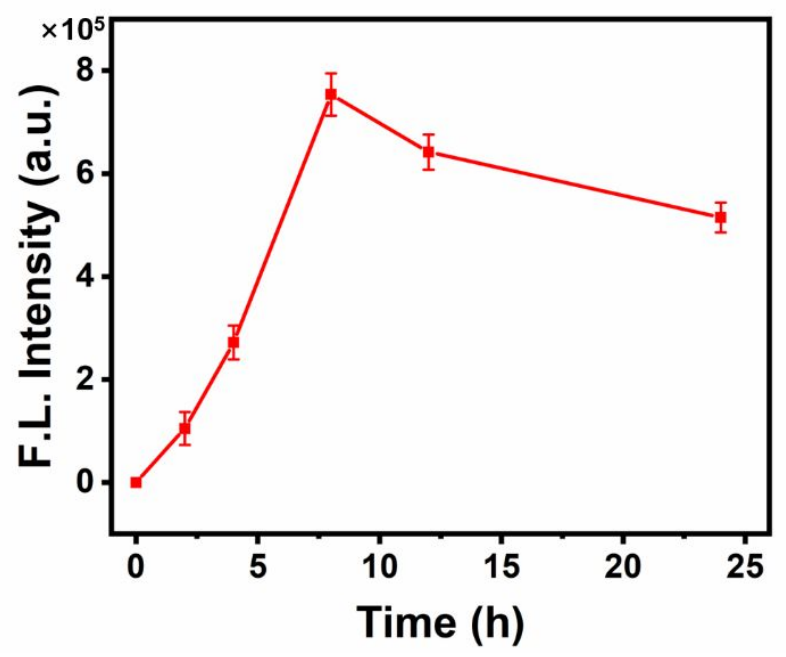

Figure S7. (a) Fluorescence intensity of tumor areas as function of time.

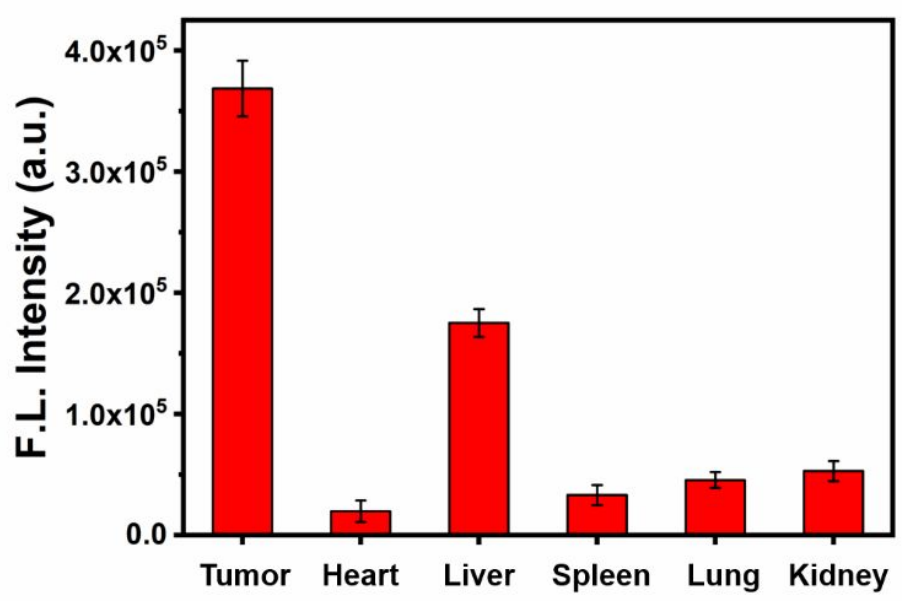

Figure S8. Fluorescence signals of HMCP NCs in tumor and major tissues. 


\section{Supporting Information}

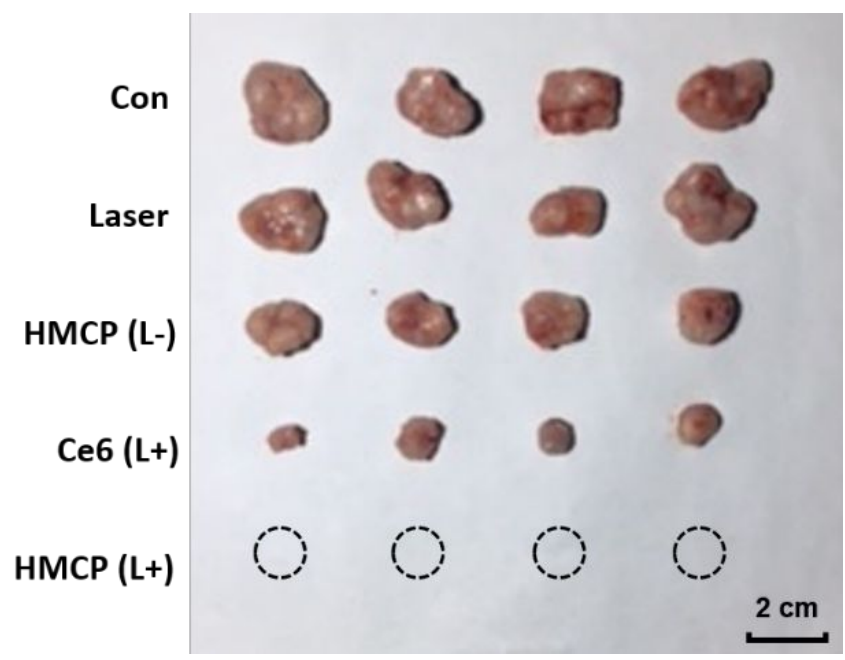

Figure S9. Digital photos of tumors after CDT/PDT combined therapy.

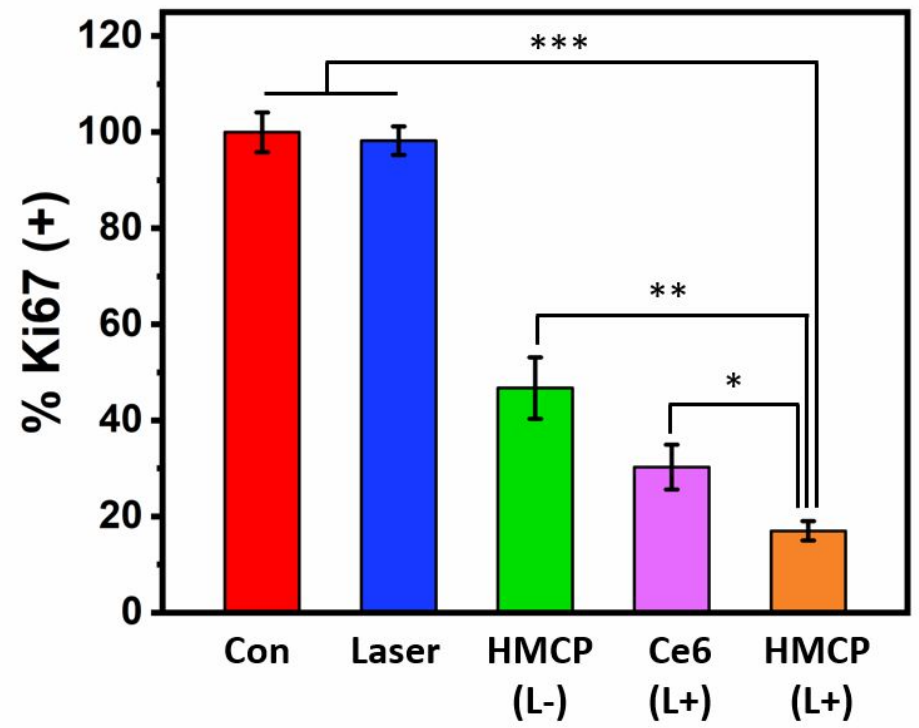

Figure S10. Statistical analysis of the Ki67 positive tumor cells. $n=4$ for panels $b$ and $d . n=$ 3 for panel g. $* \mathrm{p}<0.05, * * \mathrm{p}<0.01$, and $* * * \mathrm{p}<0.001$. 
Supporting Information

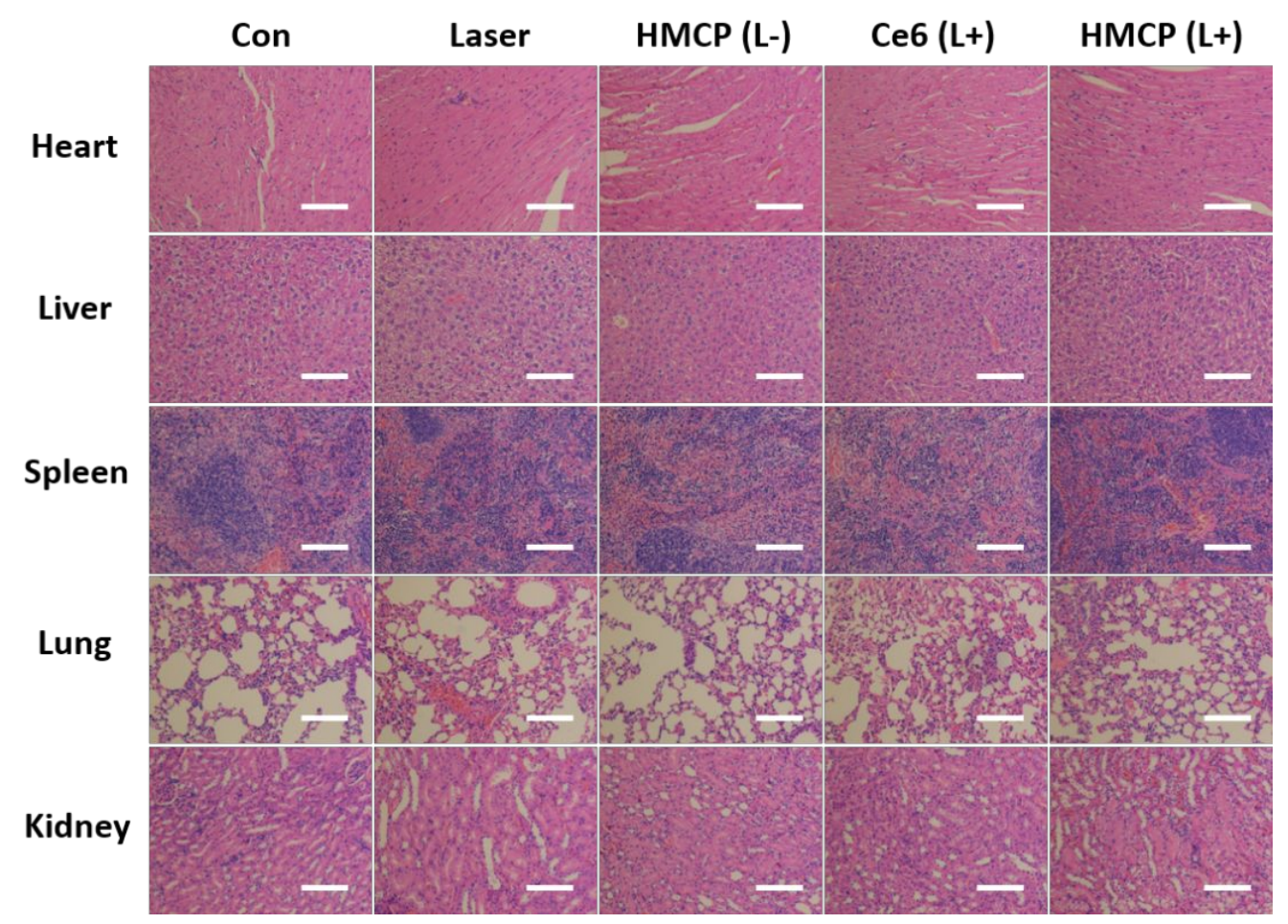

Figure S11. H\&E stain of major organs (heart, liver, spleen, lung and kidney) from mice of different groups after treatment. Scale bar: $50 \mu \mathrm{m}$. 